\title{
(i) \\ Guidance: The practical management of the gastrointestinal symptoms of pelvic radiation disease
}

\author{
H Jervoise N Andreyev, ${ }^{1}$ Ann C Muls, ${ }^{1}$ Christine Norton, ${ }^{2}$ \\ Charlotte Ralph, ${ }^{1}$ Lorraine Watson, ${ }^{1}$ Clare Shaw, ${ }^{1}$ James O Lindsay ${ }^{3}$
}

${ }^{1}$ The Gl and Nutrition Team, The Royal Marsden NHS Foundation Trust, London and Surrey, UK

${ }^{2}$ King's College London, London, UK

${ }^{3}$ Digestive Diseases Clinical Academic Unit, Barts and The London School of Medicine and Dentistry, Queen Mary University of London, London, UK

\section{Correspondence to}

Dr H J N Andreyev, The GI Unit, The Royal Marsden NHS Foundation Trust, Fulham Rd, London SW3 6JJ, UK; j@ andreyev.demon.co.uk

Received 10 April 2014 Accepted 27 April 2014 Published Online First 17 June 2014

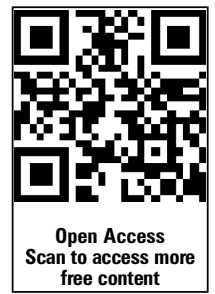

CrossMark

To cite: Andreyev HJN, Muls AC, Norton C, et al. Frontline Gastroenterology 2015:6:53-72.

\begin{abstract}
Background A recent randomised trial suggested that an algorithmic approach to investigating and managing gastrointestinal symptoms of pelvic radiation disease (PRD) is beneficial and that specially trained nurses can manage patients as effectively as a gastroenterologist.
\end{abstract}

Aims The aim of the development and peer review of the guide was to make the algorithm used in the trial accessible to all levels of clinician.

Methods Experts who manage patients with PRD were asked to review the guide, rating each section for agreement with the recommended measures and suggesting amendments if necessary. Specific comments were discussed and incorporated as appropriate, and this process was repeated for a second round of review.

Results 34 gastroenterologists, 10 nurses, 9 dietitians, 7 surgeons and 5 clinical oncologists participated in round one. Consensus (defined prospectively as $60 \%$ or more panellists selecting 'strongly agree' or 'agree') was reached for 27 of the original 28 sections in the guide, with a median of $75 \%$ of panellists agreeing with each section. $86 \%$ of panellists agreed that the guide was acceptable for publication or acceptable with minor revisions. 55 of the original 65 panellists participated in round two. $89 \%$ agreed it was acceptable for publication after the first revision. Further minor amendments were made in response to round two.

Conclusions Development of the guide in response to feedback included

- improvement of occasional algorithmic steps

- a more user-friendly layout

- clearer timeframes for referral to other teams

- expansion of reference list

- addition of procedures to the appendix.

\section{INTRODUCTION}

This guide is designed mainly to aid clinical nurse specialists looking after patients with pelvic radiation disease (PRD) working in conjunction with a gastroenterologist. However, it might also help general practitioners and generalists in investigating and treating the gastrointestinal symptoms of patients following pelvic radiotherapy.

This guide defines best practice although not every investigation modality or treatment will be available in every trust.

Those using the guide, especially if non-medically qualified, should identify a senior gastroenterologist or other appropriately qualified and experienced professionals whom they can approach easily for advice if they are practising in an unsupervised clinic.

Practitioners should not use this guide outside the scope of their competency and must identify from whom they will seek advice about abnormal test results, which they do not fully understand before they start using the guide.

Where it is stated that 'this is an emergency', the user of this guide must discuss the issue with a suitably qualified person for immediate action.

Managing patients with PRD requires a different approach to those with other forms of bowel pathology. The guide also identifies test findings that may indicate that the underlying situation is potentially serious and that advice needs to be sought urgently.

Specific therapies are usually not listed by name but as a 'class' of potential drugs as different clinicians may have local constraints or preferences as to the medications available. 
Important principles to consider when using the algorithms are

- Patients may have up to 22 gastrointestinal (GI) symptoms after pelvic radiotherapy simultaneously.

- Each symptom may have more than one cause.

- Symptoms must be investigated systematically, otherwise causes may be missed.

- Arranging all investigations at the first consultation reduces follow-up and allows directed treatment at all causes for symptoms at the earliest opportunity.

- Patients who have had radiotherapy need a different approach to patients who have GI symptoms for other reasons.

- Specialist centres only very rarely reach a new diagnosis of 'irritable bowel syndrome' in this patient group.

- Endoscopic or surgical intervention in tissues exposed to radiotherapy carries increased risk of serious complications.

This guide has three parts:

1. Introduction, guide to blood tests, how to use the algorithm and taking a history.

2. An algorithm detailing the individual investigation and treatment of each of the 22 symptoms identified as particularly relevant to this patient group.

3. A brief description of the diagnosis, treatment and management techniques of common conditions found in patients with PRD.

\section{GUIDELINE FOR BLOOD TESTS USED WITHIN THE GUIDE}

Routine:

Full blood count, urea and electrolytes, liver function, glucose, calcium

\begin{tabular}{|c|c|c|}
\hline $\begin{array}{l}\text { Haemoglobin } \\
<80 \mathrm{~g} / \mathrm{L}\end{array}$ & $\rightarrow$ & $\begin{array}{l}\text { If } \mathrm{Hb}<80 \mathrm{~g} / \mathrm{L} \text { : consider blood transfusion (checking } \\
\text { ferritin, transferrin saturation, RBC folate and } \\
\text { vitamin B12 before transfusion). } \\
\text { If iron deficient: consider iron supplements. } \\
\text { If unexplained: consider OGD and colonoscopy/CT } \\
\text { pneumocolon. }\end{array}$ \\
\hline $\begin{array}{l}\text { Anaemic } \\
\text { but } \mathrm{Hb} \\
>80 \mathrm{~g} / \mathrm{L}\end{array}$ & $\rightarrow$ & $\begin{array}{l}\text { Check ferritin, transferrin saturation, RBC folate and } \\
\text { vitamin B12. Replace if necessary, monitor } \\
\text { response. If unexplained consider OGD and } \\
\text { colonoscopy. }\end{array}$ \\
\hline $\begin{array}{l}\text { Abnormal } \\
\text { urea, } \\
\text { electrolytes }\end{array}$ & $\rightarrow$ & $\begin{array}{l}\text { Discuss with supervising clinician within } 24 \mathrm{~h} \text {. } \\
\text { Consider appropriate IV fluid therapy/oral } \\
\text { replacement. }\end{array}$ \\
\hline $\begin{array}{l}\text { Abnormal liver } \\
\text { function tests }\end{array}$ & $\rightarrow$ & $\begin{array}{l}\text { Discuss with supervising clinician within } 24 \mathrm{~h} \text {. } \\
\text { Patient will need a liver ultrasound and liver screen } \\
\text { including hepatitis B and C serology, ferritin, } \alpha \text { feta } \\
\text { protein, } \alpha 1 \text { antitrypsin, liver autoantibodies, total } \\
\text { Igs, cholesterol, triglycerides. }\end{array}$ \\
\hline $\begin{array}{l}\text { Abnormal } \\
\text { glucose level }\end{array}$ & $\rightarrow$ & $\begin{array}{l}\text { If no history of diabetes: } \\
\text { Between } 7-11 \mathrm{mmol} / \mathrm{L} \text { : refer to GP. } \\
>11 \mathrm{mmol} / \mathrm{L} \text { and ketones in urine: this is an } \\
\text { emergency. } \\
>11-20 \mathrm{mmol} \text { and no ketones in urine: discuss } \\
\text { with supervising clinician within } 24 \mathrm{~h} \text {. }\end{array}$ \\
\hline
\end{tabular}

- >20 mmol/L and no ketones in urine: this is an emergency.

If known diabetic:

- Do not check glucose levels.

- Consider checking HbA1c.

Abnormal $\rightarrow$ If $2.6-2.9 \mathrm{mmol} / \mathrm{L}$ : discuss with supervising clinician corrected within $24 \mathrm{~h}$.

calcium level $\quad$ If $>3.0 \mathrm{mmol} / \mathrm{L}$ : this is an emergency.

GP, general practitioner; RBC, red blood cell; OGD,

oesophago-gastroduodenoscopy.

Additional blood tests are indicated depending on the presenting GI symptoms and differential diagnoses as outlined in the algorithm. They potentially include ESR, CRP, red cell folate, iron studies, vitamin B12, thyroid function test, coeliac serology (TTG $\lg A)$, magnesium

\begin{tabular}{|c|c|c|}
\hline Elevated ESR/CRP & $\rightarrow$ & $\begin{array}{l}\text { Consider the following possibilities: } \\
\text { Infection (including SIBO). } \\
\text { Inflammation (including IBD). } \\
\text { Recurrent malignancy. } \\
\text { Non-GI causes (eg, rheumatoid } \\
\text { arthritis, vasculitis, connective tissue } \\
\text { disorders). }\end{array}$ \\
\hline RBC folate deficiency & $\rightarrow$ & $\begin{array}{l}\text { Consider referral to dietitian for } \\
\text { specialist dietetic advice/ } \\
\text { supplementation. }\end{array}$ \\
\hline $\begin{array}{l}\text { Iron deficiency: ferritin, \% } \\
\text { transferrin saturation, red cell } \\
\text { indices }\end{array}$ & $\rightarrow$ & $\begin{array}{l}\text { If iron is low, discuss with supervising } \\
\text { clinician and oncology team within } \\
2 \text { weeks. } \\
\text { If intolerant of oral iron: consider IV } \\
\text { iron infusion. }\end{array}$ \\
\hline If excess iron & $\rightarrow$ & $\begin{array}{l}\text { Consider haemochromatosis: discuss } \\
\text { with supervising clinician and } \\
\text { consider genetic testing. }\end{array}$ \\
\hline Low vitamin B12 & $\rightarrow$ & $\begin{array}{l}\text { Exclude possibility of inadequate } \\
\text { dietary intake-if this is the probable } \\
\text { cause, consider trial of oral vitamin } \\
\text { B12 supplements. } \\
\text { Consider possibility of pernicious } \\
\text { anaemia_-check parietal cell } \\
\text { antibody. } \\
\text { Exclude SIBO (page 17). Recheck } \\
\text { result after treatment with antibiotics. } \\
\text { If confirmed on repeat testing and } \\
\text { not treatable with oral replacement, } \\
\text { ask GP to arrange lifelong } \\
\text { intramuscular replacement. }\end{array}$ \\
\hline $\begin{array}{l}\text { Abnormal thyroid function } \\
\text { tests }\end{array}$ & $\rightarrow$ & $\begin{array}{l}\text { If TSH suppressed (<0.5 mIU/L), } \\
\text { recheck result with thyroid } \\
\text { autoantibodies. } \\
\text { If TSH suppression confirmed, request } \\
\text { GP to organise/refer for radiological } \\
\text { imaging and treatment. } \\
\text { If TSH elevated (>4.0 mIU/L). } \\
\text { Re-check result. Also check morning } \\
\text { cortisol if Na } \leq 135 \text { mmol/L and K } \\
>4 \text { mmol/L or raised urea or } \\
\text { creatinine. } \\
\text { If TSH elevation confirmed: start } \\
\text { thyroid replacement medication. } \\
\text { Request GP monitor long term. } \\
\text { Review bowel function after } \\
6 \text { - } 8 \text { weeks. }\end{array}$ \\
\hline
\end{tabular}

possibilities: arthritis, vasculitis, connective tissue specialist dietetic advice/ d oncology team within 2 weeks.

If intolerant of oral iron: consider IV with supervising clinician and senetic testing. dietary intake if this is the probable cause, consider trial of oral vitamin B12 supplements.

Consider possibility of pernicious anaemia_check parietal cell

Exclude SIBO (page 17). Recheck not treatable with oral replacement ask GP to arrange lifelong recheck result with thyroid autoantibodies. Re-check result. Also check morning cortisolif $\mathrm{Na} \leq 135 \mathrm{mmol} / \mathrm{L}$ and $\mathrm{K}$ creatinine. 6-8 weeks. 


\begin{tabular}{|c|c|c|}
\hline Abnormal coeliac serology & $\rightarrow$ & $\begin{array}{l}\text { - If IgA deficient, request IgG coeliac } \\
\text { screen. } \\
\text { - If TTG elevated, confirm with } \\
\text { duodenal biopsy. } \\
\text { - Refer for specialist dietetic advice. }\end{array}$ \\
\hline Serum Mg2+ & $\rightarrow$ & $\begin{array}{l}\text { If }<0.3 \mathrm{mmol} / \mathrm{L} \text { : this is an } \\
\text { emergency. } \\
\text { If } 0.3-0.5 \text {, consider IV replacement if } \\
\text { symptomatic or fall in Mg2 level has } \\
\text { been acute. If oral replacement is } \\
\text { given, check for response after } 5-7 \\
\text { days with repeat blood tests. } \\
\text { If oral replacement is used, } \mathrm{Mg} \text { oxide } \\
\text { or Mg aspartate provide better } \\
\text { bioavailability and cause less } \\
\text { diarrhoea than other Mg } \\
\text { preparations. }\end{array}$ \\
\hline
\end{tabular}

CRP, C-reactive protein; ESR, erythrocyte sedimentation rate; $\mathrm{Gl}$, gastrointestinal; GP, general practitioner; IBD, inflammatory bowel disease; IgA, immunoglobulin $A ;$ IgG, immunoglobulin $G$; RBC, red blood cell; SIBO, small intestinal bacterial overgrowth; TSH, thyroid stimulating hormone; TTG, tissue transglutaminase.

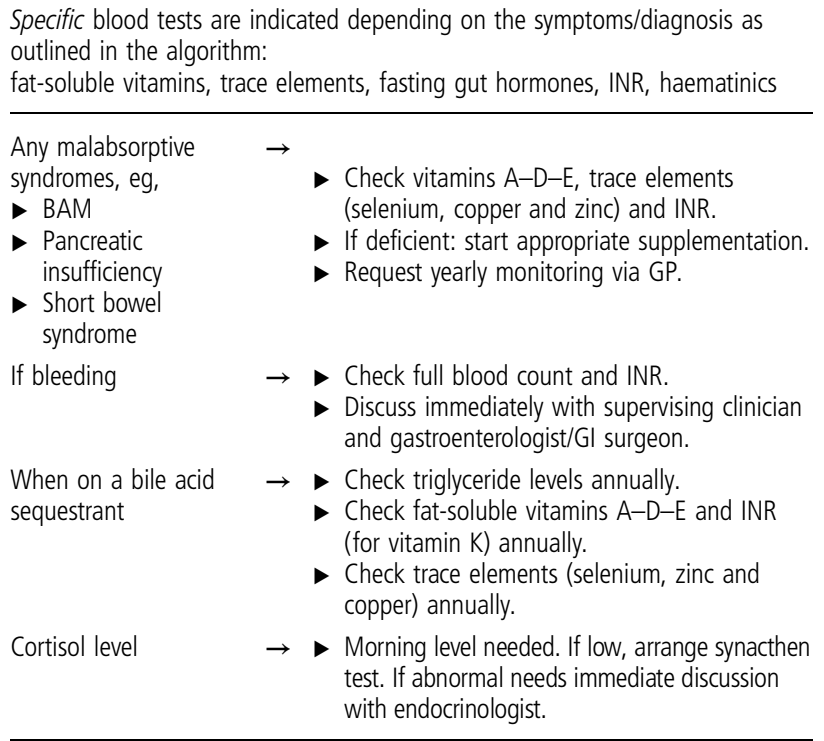

BAM, bile acid malabsorption; GI, gastrointestinal; GP, general practitioner; INR, International Normalised Ratio.

\section{HOW TO USE THE ALGORITHM}

1. Identify the symptoms by systematic history taking.

2. Examine the patient appropriately.

3. Use the algorithm to plan investigations for troublesome/ severe symptoms.

4. Most patients have more than one symptom and so investigations need to be requested for each symptom.
5. Usually all investigations are ordered at the same time and the patient reviewed with all the results.

6. When investigations should be ordered sequentially, the algorithm indicates this by stating first line, second line, etc.

7. Treatment options are generally offered sequentially but clinical judgement should be used.

\section{TAKING AN APPROPRIATE HISTORY}

Patients cannot be helped without an accurate history being taken.

- Taking a history of GI symptoms is a skill that must be learnt.

- Tools such as a Bristol Stool Chart can often clarify exactly what patients mean.

- Specialist units find that symptom questionnaires completed by the patient before the consultation often help clarify which issues are really troubling the patient.

Taking a history needs to elicit:

- What was bowel function like before the cancer emerged?

- How have the symptoms changed over time?

- Are key features indicative of reversible underlying pathology present, for example,

- Steatorrhoea?

- Nocturnal waking to defecate?

- Rapid progressive worsening of symptoms?

- Rapid weight loss?

- Has the patient noticed any masses?

- Patients and clinicians alike often miss the presence of intermittent steatorrhoea-ask:

- Is there an oily film in the lavatory water?

- Is the stool ever pale/putty-like/foul smelling/difficult to flush/floating?

A very clear definition of what a patient means when they use specific terms-for example, 'diarrhoea'/'loose stool'-what type on the Bristol Stool Chart?; 'frequency'-true bowel opening or tenesmus and incomplete evacuation?

- Is there a consistent impact of a specific component of diet on their symptoms, especially

- Fibre: how much are they eating - too much/too little?

- Fat: does this promote type 6-7 stool/steatorrhoea?

- Lactose-containing foods?

- Gluten-containing foods?

- Alcohol intake?

- Is there an association between the start of specific medication or increase in its dose and their symptoms-for example, metformin, proton pump inhibitor, $\beta$-blockers? 


\section{GI SYMPTOMS}

Bleeding (rectal): bright red \pm clots

\begin{tabular}{|c|c|c|}
\hline Investigations & Potential results & Clinical management plan: abnormal results \\
\hline $\begin{array}{l}\text { Check haemoglobin, RBC } \\
\text { indices and platelets } \\
\text { Check clotting and haematinics } \\
\text { if heavy bleeding has occurred }\end{array}$ & Abnormal & Follow treatment for abnormal blood results (pages 2-3). \\
\hline \multirow[t]{8}{*}{ Flexible sigmoidoscopy } & $\begin{array}{l}\text { Radiation proctopathy with bleeding } \\
\text { from telangiectasia }\end{array}$ & $\begin{array}{l}\text { 1. Do not biopsy irradiated areas. } \\
\text { 2. Optimise bowel function and stool consistency. } \\
\text { 3. If bleeding is not affecting quality of life, reassure. } \\
\text { 4. If bleeding affects quality of life, stop/reduce anti-coagulants if possible and } \\
\text { consider sucralfate enemas (page 19). } \\
\text { 5. Discuss referral to a specialist centre for treatment to ablate telangiectasia } \\
\text { (pages 18-19): } \\
\text { a. hyperbaric oxygen therapy } \\
\text { b. intra-rectal formalin } \\
\text { b. thermal therapy, eg, APC } \\
\text { 6. Consider referral to a specialist centre for experimental therapy within the } \\
\text { context of a clinical trial: thalidomide, vitamin A, tranexamic acid, RFA. }\end{array}$ \\
\hline & Haemorrhoidal bleeding & $\begin{array}{l}\text { If not affecting quality of life, reassure. } \\
\text { Consider local treatment of haemorrhoids (diet, topical creams). } \\
\text { Consider surgical referral for 3rd degree haemorrhoids. }\end{array}$ \\
\hline & Primary inflammatory bowel disease & $\begin{array}{l}\text { Send stool culture. If mild or moderate, refer within } 2 \text { weeks to a } \\
\text { gastroenterologist. } \\
\text { If severe, this is an emergency-discuss immediately with a } \\
\text { gastroenterologist. }\end{array}$ \\
\hline & Diverticular bleeding & $\begin{array}{l}\text { This is an emergency } \\
\text { Discuss immediately with a Gl surgeon }\end{array}$ \\
\hline & Viral infection (eg, CMV) & $\begin{array}{l}\text { This is an emergency } \\
\text { Discuss immediately with a gastroenterologist. }\end{array}$ \\
\hline & $\begin{array}{l}\text { Newly diagnosed neoplasia second } \\
\text { primary/tumour recurrence/advanced } \\
\text { polyp }\end{array}$ & $\begin{array}{l}\text { Refer urgently to the appropriate oncology team requesting an appointment } \\
\text { within } 2 \text { weeks. }\end{array}$ \\
\hline & If all tests are negative, but & 1. Consider colonoscopy. \\
\hline & symptoms persist & $\begin{array}{l}\text { 2. Optimise bowel function and stool consistency. } \\
\text { 3. Reassure and request GP to check } \mathrm{Hb} \text { as clinically indicated. }\end{array}$ \\
\hline
\end{tabular}

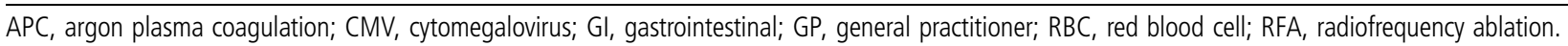




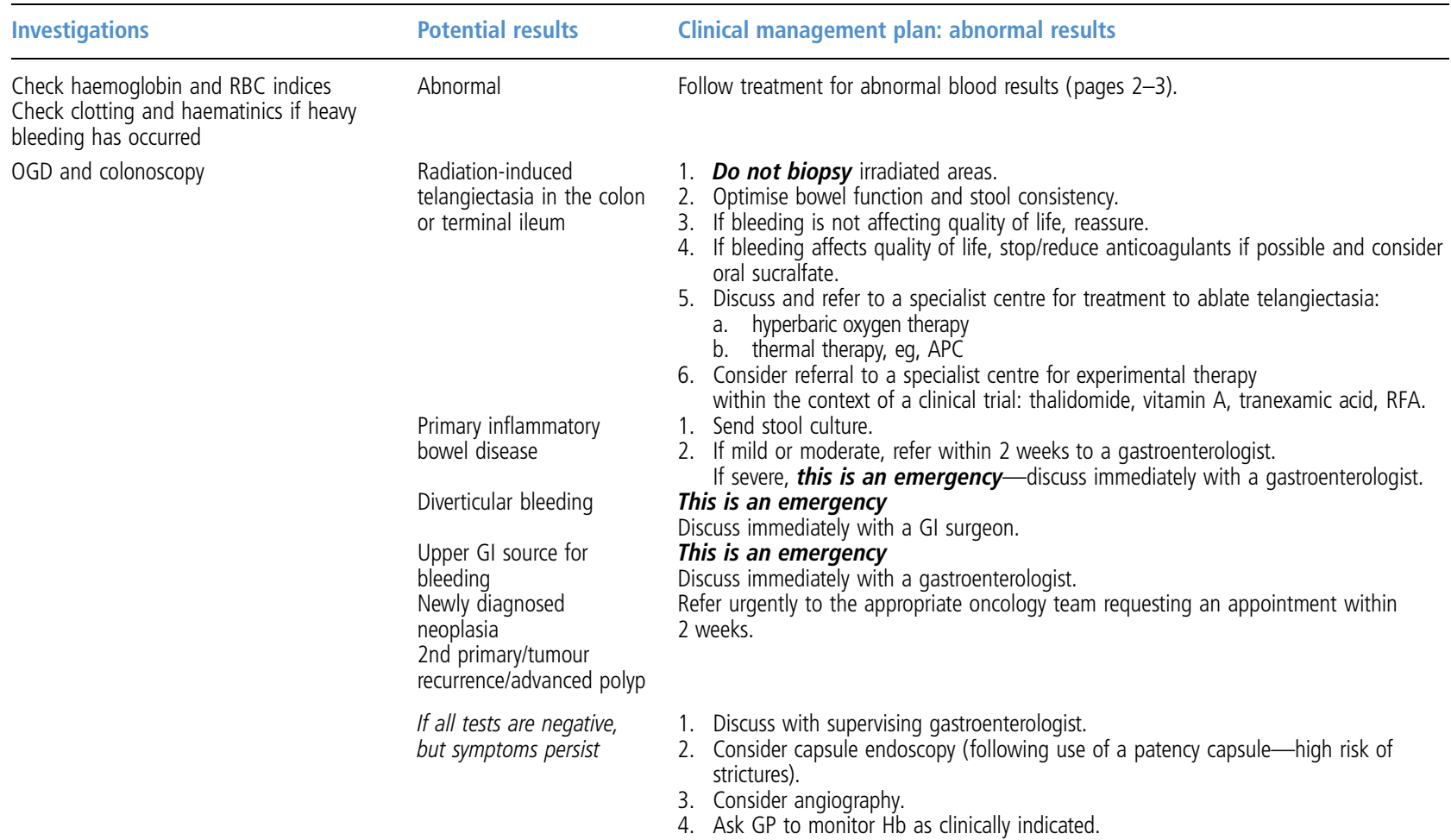

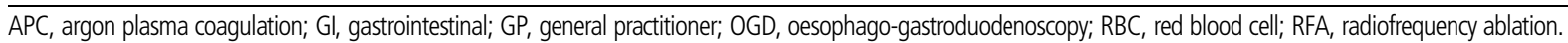

\section{Bloating/abdominal cramps}

\begin{tabular}{|c|c|c|}
\hline Investigations & Potential results & Clinical management plan: abnormal results \\
\hline \multicolumn{3}{|l|}{ 1st Line } \\
\hline $\begin{array}{l}\text { Routine AND } \\
\text { additional blood screen (pages 2-3) }\end{array}$ & Abnormal results & Follow treatment of abnormal blood results (pages 2-3). \\
\hline Abdominal X-ray & Severe faecal loading & $\begin{array}{l}\text { 1. Full bowel clearance, ie, Picolax (Ferring Pharmaceuticals Ltd, West Drayton, UK), } \\
\text { Klean-Prep (Norgine Limited, Harefield, UK), Moviprep (Norgine Limited, Harefield, UK) } \\
\text { 2. Maintenance bulk laxative. } \\
\text { 3. Correct positioning on lavatory and pelvic floor exercises (page 18). }\end{array}$ \\
\hline Dietary history & $\begin{array}{l}\text { Inadequate fluid Inadequate/ } \\
\text { excessive fibre intake } \\
\text { Excessive sorbitol } \\
\text { Excessive caffeine }\end{array}$ & $\begin{array}{l}\text { 1. Dietary advice. } \\
\text { 2. Referral to dietitian and ask patient to complete } 7 \text { day dietary diary. }\end{array}$ \\
\hline Drug history/medications assessment & & $\begin{array}{l}\text { Consider stopping opiate drugs/metformin/statins/non-steroidal anti-inflammatory } \\
\text { drugs. }\end{array}$ \\
\hline \multicolumn{3}{|r|}{ fings. } \\
\hline $\begin{array}{l}\text { OGD and duodenal aspirate and/or glucose } \\
\text { hydrogen methane breath test }\end{array}$ & SIBO & Treatment for SIBO (page 17). \\
\hline Stool for faecal elastase & EPI & Treatment for EPI (page 16). \\
\hline $\begin{array}{l}\text { Dietary history } \\
\pm \text { challenge test for carbohydrate } \\
\text { malabsorption }\end{array}$ & Carbohydrate intolerance & Treatment for carbohydrate malabsorption (pages 16-17). \\
\hline $\begin{array}{l}\text { Ultrasound of biliary tree and abdomen and } \\
\text { pelvis (and small bowel if no CT scan of } \\
\text { abdomen and pelvis in the time symptoms } \\
\text { have been present/last } 3 \text { months) }\end{array}$ & $\begin{array}{l}\text { Suggestive of gallstones, } \\
\text { inflammatory bowel disease, } \\
\text { tumour recurrence, other }\end{array}$ & $\begin{array}{l}\text { Discuss with supervising clinician and refer as clinically appropriate to a GI surgeon/ } \\
\text { gastroenterologist/oncology team. }\end{array}$ \\
\hline \multirow[t]{2}{*}{ MRI small bowel } & Small bowel stenosis & $\begin{array}{l}\text { Discuss with supervising clinician and refer as clinically appropriate to a Gl surgeon/ } \\
\text { gastroenterologist/oncology team. }\end{array}$ \\
\hline & $\begin{array}{l}\text { If all tests are negative, but } \\
\text { symptoms persist }\end{array}$ & $\begin{array}{l}\text { 1. Reassure. } \\
\text { 2. Antispasmodics. } \\
\text { 3. Low-dose antidepressants. } \\
\text { 4. Consider referral for low FODMAPs diet. } \\
\text { 5. Agent for neuropathic pain if pain severe. } \\
\text { 6. Refer to pain clinic if pain severe. } \\
\text { 7. Consider a referral for acupuncture. } \\
\text { 8. Consider a referral for hypnotherapy. }\end{array}$ \\
\hline
\end{tabular}




\section{Borborygmi}

(A rumbling/gurgling noise produced by the movement of fluid or gas through the intestine)

\begin{tabular}{|c|c|c|}
\hline Investigations & Potential results & Clinical management plan: abnormal results \\
\hline \multicolumn{3}{|l|}{ 1st Line } \\
\hline $\begin{array}{l}\text { Routine AND additional blood screen } \\
\text { (pages 2-3) }\end{array}$ & Abnormal results & Follow treatment of abnormal blood results (pages 2-3). \\
\hline $\begin{array}{l}\text { OGD and duodenal aspirate and biopsies and/ } \\
\text { or glucose hydrogen methane breath tests }\end{array}$ & $\begin{array}{l}\text { SIBO } \\
\text { Enteric infection }\end{array}$ & $\begin{array}{l}\text { Treatment for SIBO (page 17). } \\
\text { Treat as recommended by microbiologist. }\end{array}$ \\
\hline Carbohydrate challenge & Carbohydrate malabsorption & Treatment for carbohydrate malabsorption (pages 16-17). \\
\hline \multicolumn{3}{|c|}{ If borgorygmi are present in combination with other symptoms: flushing, abdominal pain, diarrhoea, wheezing, tachycardia or fluctuation in $B P$} \\
\hline \multirow{2}{*}{$\begin{array}{l}\text { Fasting gut hormones } \\
+ \text { Urinary } 5-H I A A+C T \text { scan chest, abdomen } \\
\text { and pelvis }\end{array}$} & $\begin{array}{l}\text { Functioning NET, eg, carcinoid } \\
\text { syndrome or pancreatic NET }\end{array}$ & $\begin{array}{l}\text { Discuss and refer urgently to the appropriate neuroendocrine tumour } \\
\text { team requesting an appointment within } 2 \text { weeks. }\end{array}$ \\
\hline & $\begin{array}{l}\text { If all tests are negative, but } \\
\text { symptoms persist }\end{array}$ & Reassure. \\
\hline
\end{tabular}

\section{Constipation/difficulty evacuating rectum}

\begin{tabular}{|c|c|c|}
\hline Investigations & Potential results & Clinical management plan: abnormal results \\
\hline Dietary/lifestyle/medications assessment & $\begin{array}{l}\text { Inadequate fibre intake } \\
\text { Reduced general exercise } \\
\text { Drug induced, eg, } \\
\text { opioid } \\
\text { ondanestron } \\
\text { antimuscarinic } \\
\text { loperamide } \\
\text { iron supplement } \\
\text { Chronic constipation/ } \\
\text { evacuation disorder }\end{array}$ & $\begin{array}{l}\text { 1. Dietary advice about healthy fibre and fluid intake. } \\
\text { 2. Lifestyle advice about daily exercise. } \\
\text { 3. Making time to have a toileting routine, correct positioning on } \\
\text { the lavatory. } \\
\text { 4. Medications advice. } \\
\text { 5. Rectal evacuant (eg, glycerine suppositories). } \\
\text { 6. Bulk laxative } \pm \text { rectal evacuant. } \\
\text { 7. Consider referral for biofeedback therapy (page 18). } \\
\text { 8. Consider use of probiotics. }\end{array}$ \\
\hline Abdominal/rectal examination & Anal fissure & $\begin{array}{l}\text { 1. Topical healing agent, eg, GTN or diltiazem gel (for } 8 \text { weeks). } \\
\text { 2. Stool bulking/softening agent } \pm \text { short term topical local } \\
\text { anaesthetic. } \\
\text { 3. If recurrent, consider referral for botulinum toxin treatment. } \\
\text { 4. If fissure not healed after } 2 \text { months, refer for surgical opinion. }\end{array}$ \\
\hline $\begin{array}{l}\text { Routine blood screen and additional blood } \\
\text { screen }\end{array}$ & $\begin{array}{l}\text { Dehydration } \\
\text { Hypothyroidism } \\
\text { Elevated calcium }\end{array}$ & $\begin{array}{l}\text { Encourage oral fluid intake. } \\
\text { 1. Repeat thyroid function test. } \\
\text { 2. Inform GP and follow management (pages 2-3). } \\
\text { Follow management (page } 2 \text { ). }\end{array}$ \\
\hline Abdominal X-ray & $\begin{array}{l}\text { Faecal loading/faecal } \\
\text { impaction }\end{array}$ & $\begin{array}{l}\text { 1. Full bowel clearance, eg, Picolax, Klean-Prep. } \\
\text { 2. Maintenance bulk laxative. } \\
\text { 3. Correct positioning on lavatory and pelvic floor exercises } \\
\text { (page 18). }\end{array}$ \\
\hline Transit study & Slow Gl transit & Discuss and refer to a gastroenterologist routinely. \\
\hline $\begin{array}{l}\text { Colonoscopy/CT pneumocolon if new onset } \\
\text { Flexible sigmoidoscopy for longstanding } \\
\text { problems }\end{array}$ & $\begin{array}{l}\text { Newly diagnosed neoplasm } \\
\text { Newly diagnosed IBD }\end{array}$ & $\begin{array}{l}\text { Discuss and refer to oncology team, requesting appointment within } \\
2 \text { weeks. } \\
\text { If mild or moderate, refer within } 2 \text { weeks to a gastroenterologist. } \\
\text { If severe, this is an emergency_discuss immediately with a } \\
\text { gastroenterologist. } \\
\text { Discuss with supervising clinician. } \\
\text { 1. Pelvic floor exercises (page 18). } \\
\text { 2. Bulking agent. } \\
\text { 3. Antidiarrhoeal medication. } \\
\text { 4. Low-dose tricyclic/SSRI antidepressant. } \\
\text { 5. Consider referral for sacral nerve/tibial nerve stimulation. } \\
\text { 6. Consider referral to a Gl surgeon for stoma formation. }\end{array}$ \\
\hline
\end{tabular}

$\overline{\mathrm{Gl}}$, gastrointestinal; GP, general practitioner; GTN, glyceril trinitrate; IBD, inflammatory bowel disease. 
Diarrhoea (stool type 6-7 Bristol Stool Chart)

Also use this section if patient has 'frequency of defecation', 'nocturnal defecation' or 'urgency of defecation'

\begin{tabular}{|c|c|c|}
\hline Investigations & Potential results & Clinical management plan: abnormal results \\
\hline Dietary/ lifestyle/ medications assessment & $\begin{array}{l}\text { High dietary fat intake } \\
\text { Low/high fibre intake } \\
\text { High fizzy drink intake } \\
\text { High use of sorbitol-containing } \\
\text { chewing gum or sweets } \\
\text { High caffeine intake } \\
\text { High alcohol intake } \\
\text { Anxiety } \\
\text { Drug induced, eg, } \\
\text { PPIs } \\
\text { Laxatives } \\
\beta \text { blockers } \\
\text { Metformin }\end{array}$ & $\begin{array}{l}\text { 1. Dietary advice about healthy fibre and dietary fat intake. } \\
\text { 2. Referral to dietitian and ask patient to complete 7-day } \\
\text { dietary diary beforehand. } \\
\text { 3. Lifestyle advice about smoking cessation. } \\
\text { 4. Consider referral for psychological support. } \\
\text { 5. Medications advice. } \\
\text { 6. Antidiarrhoeal } \pm \text { bulk laxative. }\end{array}$ \\
\hline
\end{tabular}

Routine AND additional blood screen (pages 2-3)

Abnormal results

$\mathrm{Mg}^{2+}$ low

Coeliac disease

Stool sample: for microscopy, culture and Clostridium difficile toxin

Stool sample: for faecal elastase

Stool contains pathogen

OGD with duodenal aspirate and biopsies and/or glucose hydrogen (methane) breath test

Carbohydrate challenge

SeHCAT scan

Abdominal $X$-ray

\section{1st Line}

Flexible sigmoidoscopy with biopsies from non-irradiated bowel (avoid biopsies from areas obviously irradiated in sigmoid and rectum)

EPI

SIBO BAM frequency of defecation
Specific disaccharide intolerance

Faecal loading with overflow

Radiation proctopathy and

Radiation proctopathy/colopathy and pelvic floor dysfunction (page 17)

Macroscopic colitis

Microscopic colitis

\section{2nd Line}

Colonoscopy with biopsies

If diarrhoea is present in combination with other symptoms: flushing, abdominal pain, borborygmi, wheezing, tachycardia or fluctuation in BP

\section{3rd Line}

Gut hormones (Chromogranin A\&B, gastrin, substance P, VIP, calcitonin, somatostatin, pancreatic polypeptide) and Urinary 5-HIAA and CT chest, abdomen and pelvis
Functioning NET, eg, carcinoid syndrome or pancreatic NET

If all tests are negative, but symptoms persist
Follow treatment of abnormal blood results (pages 2-3).

1. If IgA deficient, request IgG coeliac screen.

2. Confirm with duodenal biopsy.

3. Refer to dietitian for gluten free diet.

4. Liaise with GP regarding long term monitoring of bone densitometry and referral to a coeliac clinic.

Treat as recommended by the microbiologist and local protocols.

See EPI (page 16)

Treatment for SIBO (page 17).

Appropriate treatment (pages 16-17).

Treatment for BAM (page 16).

Bulking agent.

1. Pelvic floor and toileting exercises (page 18) min. 6 weeks.

2. Add stool bulking agent to pelvic floor exercise regimen.

3. Antidiarrhoeal \pm stool bulking agent.

1. Antidiarrhoeal.

2. \pm stool bulking agent.

3. \pm pelvic floor and toileting exercises (page 18).

1. Send stool culture.

2. If mild or moderate, refer within 2 weeks to a gastroenterologist. If severe, this is an emergencydiscuss immediately with a gastroenterologist.

Discuss with supervising clinician and refer to a gastroenterologist.

As above.

Discuss with the appropriate clinical team within $24 \mathrm{~h}$.

Discuss and refer to the appropriate neuroendocrine tumour team requesting an appointment within 2 weeks.

Reassure and suggest symptomatic treatment with antidiarrhoeal drugs.

Trial of low-dose tricyclic antidepressants.

Biofeedback.

Note: faecal calprotectin as a marker for bowel inflammation is too non-specific and hence not recommended in this population.

BAM, bile acid malabsorption; EPI, excocrine pancreatic insufficiency; GP, general practitioner; IgA, immunoglobulin $A$; IgG, immunoglobulin G; OGD,

oesophago-gastroduodenoscopy; PPI, proton pump inhibitor; NET, neuroendocrine tumour; SIBO, small intestinal bacterial overgrowth. 
Faecal incontinence

(Soiling/leakage/using pads)

\begin{tabular}{|c|c|c|}
\hline Investigations & Potential results & Clinical management plan: abnormal results \\
\hline \multicolumn{3}{|l|}{ 1st Line } \\
\hline Routine AND additional blood screen (pages 2-3) & Abnormal results & Follow treatment of abnormal blood results (pages $2-3$ ). \\
\hline $\begin{array}{l}\text { Rectal examination Anoscopy } \\
\text { Flexible sigmoidoscopy }\end{array}$ & $\begin{array}{l}\text { Pelvic floor dysfunction (page 17) } \\
\text { with radiation proctopathy and faecal } \\
\text { incontinence/leakage OR } \\
\text { Anal sphincter defect } \\
\text { Stool consistency: type } 6-7 \\
\text { Constipation with overflow diarrhoea } \\
\text { Mucus leakage } \\
\text { Mucosal prolapse } \\
\text { Unrelated to radiotherapy (eg, } \\
\text { childbirth, previous sphincter surgery, } \\
\text { haemorrhoidectomy, idiopathic) }\end{array}$ & $\begin{array}{l}\text { 1. Pelvic floor and toileting exercises (page 18). } \\
\text { 2. Stool bulking } \pm \text { antidiarrhoeal agent. } \\
\text { 3. Antidiarrhoeal agent } \pm \text { stimulant laxative suppositories/enemas. } \\
\text { 4. Topical sympathomimetic agent (eg, phenylephrine). } \\
\text { 5. Perianal skin care (pages 19-20). } \\
\text { 6. Referral for biofeedback. } \\
\text { 7. Consider referral to a specialist centre for sacral nerve stimulation. } \\
\text { 8. Consider referral to a specialist centre for defunctioning surgery/sphincter repair. } \\
\text { See 'diarrhoea' (page 7). } \\
\text { See 'constipation' (page 6). } \\
\text { See 'mucus discharge' (page 9). } \\
\text { Routine referral to a Gl surgeon. } \\
\text { Refer to a specialist team for management of faecal incontinence. }\end{array}$ \\
\hline \multicolumn{3}{|l|}{ 2nd Line } \\
\hline $\begin{array}{l}\text { Endo anal ultrasound } \\
\text { AND } \\
\text { Anorectal physiology }\end{array}$ & $\begin{array}{l}\text { Muscular incoordination or } \\
\text { inadequate function } \\
\text { Significant sphincter defect }\end{array}$ & $\begin{array}{l}\text { Pelvic floor and toileting exercises (page 18) } \\
\text { Bulking agent (Normacol (Norgine, Harefield, UK) or loperamide. } \\
\text { Biofeedback (page 18). } \\
\text { Discuss with supervising clinician and routine referral to GI surgeon for consideration } \\
\text { of sacral nerve or tibial nerve stimulation. }\end{array}$ \\
\hline
\end{tabular}

Gl, gastrointestinal.

Flatulence (oral-burping)

\begin{tabular}{|c|c|c|}
\hline Investigations & Potential results & Clinical management plan: abnormal results \\
\hline \multicolumn{3}{|l|}{ 1st Line } \\
\hline Dietary assessment & High intake of fizzy drinks & Reduce intake of fizzy drinks and discuss alternatives. \\
\hline \multicolumn{3}{|l|}{ 2nd Line } \\
\hline Abdominal X-ray & Faecal loading & See 'constipation' (page 6). \\
\hline \multirow{2}{*}{$\begin{array}{l}\mathrm{OGD} \text { and } \mathrm{D} 2 \text { aspirate and/or glucose } \\
\text { hydrogen (methane) breath test }\end{array}$} & SIBO & Treatment for SIBO (page 17). \\
\hline & $\begin{array}{l}\text { If all tests are negative, but } \\
\text { symptoms persist }\end{array}$ & Discuss 'aerophagia' with patient. \\
\hline
\end{tabular}

OGD, oesophago-gastroduodenoscopy; SIBO, small intestinal bacterial overgrowth.

\section{Flatulence (rectal)}

\begin{tabular}{|c|c|c|}
\hline Investigations & Potential results & Clinical management plan: abnormal results \\
\hline \multicolumn{3}{|l|}{ 1st Line } \\
\hline Dietary assessment & $\begin{array}{l}\text { Excess/deficient fibre intake/resistant } \\
\text { starch } \\
\text { Inadequate fluids }\end{array}$ & $\begin{array}{l}\text { Referral to dietitian and ask patient to complete } 7 \text { day dietary diary in advance. } \\
\text { Dietitian to assess food diary to determine dietary fibre intake. } \\
\text { Give appropriate advice. }\end{array}$ \\
\hline \multicolumn{3}{|l|}{ 2nd Line } \\
\hline Abdominal X-ray & $\begin{array}{l}\text { Constipation } \\
\text { Faecal loading }\end{array}$ & $\begin{array}{l}\text { See 'constipation' (page 6). } \\
\text { 1. Full bowel clearance, eg, Picolax, Klean-Prep, Moviprep. } \\
\text { 2. Maintenance bulk laxative. } \\
\text { 3. Correct positioning on lavatory and pelvic floor exercises (page 18). }\end{array}$ \\
\hline $\begin{array}{l}\text { OGD and D2 aspirate and/or glucose hydrogen (methane) } \\
\text { breath test }\end{array}$ & SIBO & Treatment for SIBO (page 17). \\
\hline Flexible sigmoidoscopy & Newly diagnosed neoplasm & $\begin{array}{l}\text { Refer urgently to the appropriate oncology team, requesting an appointment within } \\
2 \text { weeks. }\end{array}$ \\
\hline & Newly diagnosed IBD & $\begin{array}{l}\text { 1. Send stool culture. } \\
\text { 2. If mild or moderate, refer within } 2 \text { weeks to a gastroenterologist. } \\
\text { If severe, this is an emergency — discuss immediately with a gastroenterologist. }\end{array}$ \\
\hline Rectal examination & $\begin{array}{l}\text { Pelvic floor dysfunction (page 17) } \\
\text { Lax sphincter muscle }\end{array}$ & $\begin{array}{l}\text { 1. Pelvic floor and toileting exercises (page 18). } \\
\text { 2. Stool bulking } \pm \text { antidiarrhoeal agent. } \\
\text { 3. Antidiarrhoeal agent } \pm \text { stimulant laxative suppositories/enemas. } \\
\text { 4. Referral for biofeedback (page } 18 \text { ). }\end{array}$ \\
\hline
\end{tabular}

IBD, inflammatory bowel disease; OGD, oesophago-gastroduodenoscopy; SIBO, small intestinal bacterial overgrowth. 
Loss of sensation

(Unable to discriminate between need to defecate and pass urine)

\begin{tabular}{|c|c|c|}
\hline Investigations & Potential results & Clinical management plan: abnormal results \\
\hline $\begin{array}{l}\text { Neurological } \\
\text { examination } \\
\text { (including perianal } \\
\text { sensation) }\end{array}$ & $\begin{array}{l}\text { Abnormal examination (eg, } \\
\text { suspected spinal cord } \\
\text { compression, cauda equine } \\
\text { syndrome, neurogenic bladder) }\end{array}$ & $\begin{array}{l}\text { This is an emergency } \\
\text { Discuss immediately with an oncology or neurology team. }\end{array}$ \\
\hline $\begin{array}{l}\text { Routine blood } \\
\text { screen and ESR, } \\
\text { vitamin B12, red } \\
\text { cell folate }\end{array}$ & Abnormal results & Follow treatment of abnormal blood results (pages $2-3$ ). \\
\hline \multirow[t]{2}{*}{$\begin{array}{l}\text { Consider MRI } \\
\text { pelvis }\end{array}$} & $\begin{array}{l}\text { Tumour recurrence or other } \\
\text { cause for neurological } \\
\text { dysfunction }\end{array}$ & Discuss immediately with supervising clinician. \\
\hline & $\begin{array}{l}\text { Related to radiotherapy or } \\
\text { surgery }\end{array}$ & $\begin{array}{l}\text { 1. Pelvic floor and toileting exercises (page 18). } \\
\text { 2. Bulking agent } \pm \text { antidiarrhoeal. } \\
\text { 3. Consider referral for biofeedback (page 18). }\end{array}$ \\
\hline
\end{tabular}

ESR, erythrocyte sedimentation rate.

\section{Mucus discharge}

\begin{tabular}{|c|c|c|}
\hline Investigations & Potential results & Clinical management plan: abnormal results \\
\hline Dietary assessment & Excessive dietary fibre intake & $\begin{array}{l}\text { 1. Refer to dietitian for detailed dietary review and advice. } \\
\text { 2. Pelvic floor and toileting exercises (page 18). }\end{array}$ \\
\hline Rectal examination & $\begin{array}{l}\text { Haemorrhoids } \\
\text { Anal lesion } \\
\text { Rectal lesion }\end{array}$ & $\begin{array}{l}\text { Stool bulking/softening agent. } \\
\pm \text { short-term topical local anaesthetic. } \\
\text { Refer urgently to a Gl surgeon. } \\
\text { Refer for a flexible sigmoidoscopy within } 2 \text { weeks. }\end{array}$ \\
\hline Flexible sigmoidoscopy & $\begin{array}{l}\text { Anorectal ulcer } \\
\text { Neoplastic } \\
\text { Rectal mucosal prolapse } \\
\text { Traumatic ulceration/solitary rectal ulcer } \\
\text { syndrome } \\
\text { If radiation-ulceration related }\end{array}$ & $\begin{array}{l}\text { Determine patient is not on nicorandil for angina. } \\
\text { Refer urgently to the appropriate oncology team requesting } \\
\text { an appointment within } 2 \text { weeks. } \\
\text { Refer to a Gl surgeon. } \\
\text { Refer to a gastroenterologist. } \\
\text { Do not biopsy } \\
\text { 1. Sucralfate enemas. } \\
\text { 2. Consider stool bulking/softening agent. } \\
\text { 3. Antibiotics. } \\
\text { 4. Consider hyperbaric oxygen therapy. } \\
\text { 5. Refer to a specialist centre. } \\
\text { Refer for endoscopic removal. } \\
\text { Refer to the appropriate oncology team requesting an appointment } \\
\text { within } 2 \text { weeks. } \\
\text { 1. Send stool culture. } \\
\text { 2. If mild or moderate, refer within } 2 \text { weeks to } \\
\text { a gastroenterologist. } \\
\text { If severe, this is an emergency-discuss immediately with a } \\
\text { gastroenterologist. }\end{array}$ \\
\hline $\begin{array}{l}\text { OGD and D2 aspirate and/or } \\
\text { glucose } \\
\text { hydrogen (methane) breath test }\end{array}$ & $\mathrm{SIBO}$ & Treatment for SIBO (page 17). \\
\hline
\end{tabular}

GI, gastrointestinal; IBD, inflammatory bowel disease; OGD, oesophago-gastroduodenoscopy; SIBO, small intestinal bacterial overgrowth. 
Nausea and vomiting

\begin{tabular}{|c|c|c|}
\hline Investigations & Potential results & Clinical management plan: abnormal results \\
\hline Fundoscopy & Raised ICP & $\begin{array}{l}\text { This is an emergency } \\
\text { Discuss immediately with supervising clinician and the oncology or } \\
\text { neurology team. }\end{array}$ \\
\hline Trial of PPI & Inflammatory (acid related) & Reassess after 2-4 weeks as clinically indicated. \\
\hline Blood screen+morning cortisol level & $\begin{array}{l}\text { Metabolic abnormality } \\
\text { Liver/biliary abnormality } \\
\text { Suggestive of infection }\end{array}$ & $\begin{array}{l}\text { Discuss immediately with supervising clinician and consider referral to } \\
\text { endocrinology within } 24 \mathrm{~h} \text {. } \\
\text { Discuss with supervising clinician within } 24 \mathrm{~h} \text {. } \\
\text { Treat with antibiotics within level of confidence or discuss with } \\
\text { microbiologists and supervising clinician. }\end{array}$ \\
\hline Urine analysis & $\begin{array}{l}\text { Metabolic abnormality, eg, } \\
\text { glucosuria, ketonuria } \\
\text { Infection }\end{array}$ & $\begin{array}{l}\text { Discuss immediately with supervising clinician. } \\
\text { Treat with antibiotics within level of confidence or discuss with } \\
\text { microbiologists and supervising clinician within } 24 \mathrm{~h} \text {. }\end{array}$ \\
\hline $\begin{array}{l}\text { OGD } \\
\pm \text { assessment for Helicobacter pylori }\end{array}$ & Inflammatory/ulcerative disease & $\begin{array}{l}\text { 1. PPI and helicobacter eradication therapy. } \\
\text { 2. Sucralfate. } \\
\text { 3. Promotility agents. } \\
\text { 4. Discuss with supervising clinician need for future repeat } \\
\text { endoscopy. } \\
\text { Consider a prokinetic medication (page 20) (eg, domperidone, } \\
\text { metoclopramide, erythromycin). }\end{array}$ \\
\hline Glucose hydrogen (methane) breath test & SIBO & Treatment for SIBO (page 17). \\
\hline Hepatic and pancreatic ultrasound & $\begin{array}{l}\text { Biliary/hepatic/pancreatic } \\
\text { aetiology }\end{array}$ & $\begin{array}{l}\text { Discuss with gastroenterologist or hepatology team. } \\
\text { If acute jaundice/cholangitis: this is an emergency. }\end{array}$ \\
\hline \multirow[t]{2}{*}{$\begin{array}{l}\text { CXR/CT/MRI } \\
\text { (including CNS) }\end{array}$} & $\begin{array}{l}\text { Local or distal infection } \\
\text { Central nervous system } \\
\text { pathology } \\
\text { Bowel obstruction }\end{array}$ & $\begin{array}{l}\text { Treat with antibiotics within level of confidence or discuss with } \\
\text { microbiologists and supervising clinician. } \\
\text { This is an emergency } \\
\text { Discuss immediately with supervising clinician and the oncology or } \\
\text { neurology team. } \\
\text { This is an emergency } \\
\text { Discuss immediately with a GI surgeon. }\end{array}$ \\
\hline & $\begin{array}{l}\text { If all tests are negative, but } \\
\text { symptoms persist }\end{array}$ & $\begin{array}{l}\text { 1. Consider contributing psychological factors. } \\
\text { 2. Consider referral for psychological support if there is a possible } \\
\text { underlying eating disorder. } \\
\text { 3. Consider a routine referral to a gastroenterologist for further } \\
\text { management. }\end{array}$ \\
\hline
\end{tabular}

GI, gastrointestinal; OGD, oesophago-gastroduodenoscopy; PPI, ; SIBO, small intestinal bacterial overgrowth. 
Pain (abdominal)

\begin{tabular}{|c|c|c|}
\hline Investigations & Potential results & Clinical management plan: abnormal results \\
\hline \multicolumn{3}{|l|}{ 1st Line } \\
\hline Dietary assessment & $\begin{array}{l}\text { Inappropriate fluid and fibre } \\
\text { intake } \\
\text { Excessive sorbitol } \\
\text { Excessive caffeine }\end{array}$ & Dietary advice about healthy fibre and general dietary intake. \\
\hline Medication assessment & $\begin{array}{l}\text { Drug induced, eg, } \\
\text { opioid } \\
\text { ondansetron } \\
\text { anti-muscarinics } \\
\text { loperamide } \\
\text { iron supplement } \\
\text { statin } \\
\text { metformin }\end{array}$ & Medications advice. \\
\hline Routine blood tests and calcium, ESR, CRP & Abnormal results & Follow treatment of abnormal blood results (pages 2-3). \\
\hline Abdominal X-ray & $\begin{array}{l}\text { Faecal loading/faecal } \\
\text { impaction }\end{array}$ & $\begin{array}{l}\text { 1. Full bowel clearance, eg, Picolax, Klean-Prep. } \\
\text { 2. Maintenance bulk laxative. } \\
\text { 3. Correct positioning on lavatory (toileting exercises) (page 18). }\end{array}$ \\
\hline \multicolumn{3}{|l|}{ 2nd Line } \\
\hline $\begin{array}{l}\text { OGD and duodenal aspirate } \pm \text { glucose } \\
\text { hydrogen (methane) breath tests }\end{array}$ & SIBO & Treatment for SIBO (page 17). \\
\hline Flexible sigmoidoscopy & Newly diagnosed IBD & $\begin{array}{l}\text { 1. Send stool culture. } \\
\text { 2. If mild or moderate, refer within } 2 \text { weeks to a gastroenterologist. } \\
\text { If severe, this is an emergency-discuss immediately with a } \\
\text { gastroenterologist. }\end{array}$ \\
\hline $\begin{array}{l}\text { Ultrasound of biliary tree and small bowel } \\
\text { (if no recent CT scan of abdomen and pelvis) }\end{array}$ & $\begin{array}{l}\text { Suggestive of gallstones, IBD, } \\
\text { tumour recurrence, other } \\
\text { If all tests are negative, but } \\
\text { symptoms persist }\end{array}$ & $\begin{array}{l}\text { Discuss with supervising clinician within } 24 \mathrm{~h} \text { and refer as clinically } \\
\text { appropriate to a } \mathrm{Gl} \text { surgeon/gastroenterologist/oncology team. } \\
\text { 1. Consider } \mathrm{CT} \text { abdomen and pelvis. } \\
\text { 2. Consider lower } \mathrm{Gl} \text { endoscopic assessment. } \\
\text { 3. Refer to a specialist pain team for further assessment. } \\
\text { 4. Consider antispasmodics. Consider low-dose antidepressants. } \\
\text { 5. Consider agent for neuropathic pain. } \\
\text { 6. Consider referral for acupuncture. }\end{array}$ \\
\hline
\end{tabular}

CRP, C-reactive protein; ESR, erythrocyte sedimentation rate; GI, gastrointestinal; IBD, inflammatory bowel disease; OGD, oesophago-gastroduodenoscopy; SIBO, small intestinal bacterial overgrowth.

\section{Pain (back-new onset)}

\begin{tabular}{|c|c|c|}
\hline Investigations & Potential results & Clinical management plan: abnormal results \\
\hline $\begin{array}{l}\text { Neurological examination (including perianal } \\
\text { sensation) }\end{array}$ & $\begin{array}{l}\text { Abnormal examination } \\
\text { (eg, suspected spinal cord } \\
\text { compression) }\end{array}$ & $\begin{array}{l}\text { This is an emergency } \\
\text { Discuss immediately with an oncology or neurology team. }\end{array}$ \\
\hline Symptom assessment & $\begin{array}{l}\text { Pain in the lower flank } \\
\text { Pain in the lower back } \\
\text { Bone pain }\end{array}$ & $\begin{array}{l}\text { 1. Consider pyelonephritis kidney infection/stone/urinary tract } \\
\text { infection. } \\
\text { 2. Urine dip stick and urine sample for culture and sensitivity. } \\
\text { 3. Consider renal ultrasound. } \\
\text { Consider constipation, faecal loading and faecal impaction (page 6). } \\
\text { 1. Consider lower back fracture. } \\
\text { 2. Request a spinal (thoracic/lumbar) X-ray. } \\
\text { 3. Consider MRI. } \\
\text { Consider a bone scan and myeloma screen. }\end{array}$ \\
\hline $\begin{array}{l}\text { Routine blood screen and additional blood } \\
\text { screen }\end{array}$ & $\begin{array}{l}\text { Abnormal results suggesting } \\
\text { cancer relapse }\end{array}$ & Discuss immediately with supervising clinician. \\
\hline CT/MRI/PET scan abdomen and pelvis & $\begin{array}{l}\text { Colonic faecal loading } \\
\text { Acute bowel obstruction } \\
\text { Spinal fracture }\end{array}$ & $\begin{array}{l}\text { See 'constipation' (page 6). } \\
\text { This is an emergency } \\
\text { Discuss immediately with a Gl surgeon. } \\
\text { Discuss immediately with supervising clinician. }\end{array}$ \\
\hline
\end{tabular}

Gl, gastrointestinal; PET, position emission tomography. 
Pain (anal/perianal/rectal): typical proctalgia fugax

(A sudden, severe pain in the anorectal region lasting less than $20 \mathrm{~min}$, resolving spontaneously)

\begin{tabular}{lll}
\hline Investigations & Potential results & Clinical management plan: abnormal results \\
\hline Symptom assessment & Spasm of the levator ani & Treatment for rectal spasm \\
muscles & 1. Pelvic floor and toileting exercises (page 18). \\
& 2. Consider a low dose antidepressant. \\
& 3. Consider a trial of an inhaled $\beta 2$ agonist. \\
& 4. Consider referral to a specialist centre for biofeedback (page 18). \\
& 5. Consider referral for acupuncture. \\
\hline
\end{tabular}

Pain (anal/perianal/rectal): related to defecation

\begin{tabular}{|c|c|c|}
\hline Investigations & Potential results & Clinical management plan: abnormal results \\
\hline Medication assessment & On nicorandil & Liaise with cardiology team and GP to offer alternative medication. \\
\hline \multirow{3}{*}{$\begin{array}{l}\text { Visual assessment } \\
\text { 1st Line } \\
\text { Anoscopy and flexible sigmoidoscopy }\end{array}$} & Haemorrhoids & $\begin{array}{l}\text { 1. Stool bulking/softening agent } \pm \text { short-term topical local } \\
\text { anaesthetic. }\end{array}$ \\
\hline & & $\begin{array}{l}\text { 2. Consider referral for surgical review for grade } 3 \text { or } 4 \\
\text { haemorrhoids. }\end{array}$ \\
\hline & Anal fissure & 1. Topical healing agent, eg, GTN or diltiazem gel (for 8 weeks). \\
\hline \multirow[t]{8}{*}{$\begin{array}{l}\text { 2nd Line } \\
\text { MRI }\end{array}$} & & $\begin{array}{l}\text { 2. Stool bulking/softening agent } \pm \text { short-term topical local } \\
\text { anaesthetic. } \\
\text { 3. If fissure not healed after } 2 \text { months, refer for suraical opinion. }\end{array}$ \\
\hline & Anorectal fistula & $\begin{array}{l}\text { 1. Pelvic MRI. } \\
\text { 2. Refer to a colorectal surgeon. }\end{array}$ \\
\hline & Anorectal abscess & $\begin{array}{l}\text { This is an emergency } \\
\text { Discuss immediately with a colorectal surgeon regarding treatment } \\
\text { with antibiotics and/or drainage. }\end{array}$ \\
\hline & Anorectal ulcer & Check patient is not on nicorandil. \\
\hline & $\begin{array}{l}\text { Mucosal prolapse/solitary } \\
\text { rectal ulcer }\end{array}$ & Refer to GI surgeon/gastroenterologist. \\
\hline & Neoplastic ulcer & $\begin{array}{l}\text { Refer urgently to appropriate oncology team requesting an } \\
\text { appointment within } 2 \text { weeks. }\end{array}$ \\
\hline & If radiation-ulceration related & $\begin{array}{l}\text { Do not biopsy } \\
\text { 1. Sucralfate enemas. } \\
\text { 2. Consider stool bulking/softening agent. } \\
\text { 3. Antibiotics. } \\
\text { 4. Consider hyperbaric oxygen therapy. } \\
\text { 5. Refer to a specialist centre. }\end{array}$ \\
\hline & $\begin{array}{l}\text { If all tests are negative, but } \\
\text { symptoms persist }\end{array}$ & $\begin{array}{l}\text { 1. Consider investigation under anaesthesia. } \\
\text { 2. Pelvic floor and toileting exercises (page 18). } \\
\text { 3. Stool bulking agent } \pm \text { laxative. } \\
\text { 4. Consider a referral for acupuncture. } \\
\text { 5. Consider referral to a specialist pain team. } \\
\text { 6. Consider a low-dose antidepressant. } \\
\text { 7. Consider an agent for neuropathic pain. } \\
\text { 8. Consider referral for a urological/gynaecological opinion. }\end{array}$ \\
\hline
\end{tabular}

Gl, gastrointestinal; GP, general practitioner; GTN, glyceril trinitrate. 
Pruritus (perianal)

\begin{tabular}{|c|c|c|}
\hline Investigations & Potential results & Clinical management plan: abnormal results \\
\hline \multirow[t]{2}{*}{ Symptom assessment } & $\begin{array}{l}\text { Perianal pruritus mainly } \\
\text { present during the night }\end{array}$ & $\begin{array}{l}\text { 1. Consider enterobiosis (eggs are not visible with the naked eye } \\
\text { and stool samples are only positive in 5-15\%). } \\
\text { 2. Send a sample of transparent adhesive tape (eg, Scotch Tape) } \\
\text { applied on the anal area for microscopic analysis. }\end{array}$ \\
\hline & $\begin{array}{l}\text { Due to excess pancreatic } \\
\text { enzyme replacement }\end{array}$ & Alter dose. \\
\hline \multirow[t]{2}{*}{ Visual assessment } & Changes due to radiotherapy & $\begin{array}{l}\text { 1. If soiling see guidance for faecal incontinence (page 8). } \\
\text { 2. If loose stool/diarrhoea present investigate for possible causes } \\
\text { (page 7). } \\
\text { 3. Perianal skin care (pages 19-20). } \\
\text { 4. Topical barrier agent. } \\
\text { 5. Topical corticosteroids (Trimovate (GlaxoSmithKline UK, Uxbridge, } \\
\text { UK)). } \\
\text { 6. Consider referral to dermatologist. }\end{array}$ \\
\hline & $\begin{array}{l}\text { No changes due to } \\
\text { radiotherapy }\end{array}$ & $\begin{array}{l}\text { 1. Perianal skin care (pages } 19-20 \text { ). } \\
\text { 2. Consider referral to dermatologist. }\end{array}$ \\
\hline \multirow[t]{8}{*}{ Protoscopy/flexible sigmoidoscopy } & Haemorrhoids & $\begin{array}{l}\text { 1. Stool bulking/softening agent } \pm \text { short-term topical local } \\
\text { anaesthetic. } \\
\text { 2. Consider referral for surgical review for grade } 3 \text { or } 4 \\
\text { haemorrhoids. }\end{array}$ \\
\hline & Anal fissure & $\begin{array}{l}\text { 1. Topical healing agent, eg, GTN or diltiazem gel (for } 8 \text { weeks). } \\
\text { 2. Stool bulking/softening agent } \pm \text { short-term topical local } \\
\text { anaesthetic. } \\
\text { 3. If fissure not healed after } 2 \text { months, refer for surgical opinion. }\end{array}$ \\
\hline & Anorectal fistula & $\begin{array}{l}\text { 1. Pelvic MRI. } \\
\text { 2. Refer to a colorectal surgeon. }\end{array}$ \\
\hline & Anorectal abscess & $\begin{array}{l}\text { This is an emergency } \\
\text { Discuss immediately with a Gl surgeon regarding treatment with } \\
\text { antibiotics and/or drainage. }\end{array}$ \\
\hline & Anorectal ulcer & Check patient is not on nicorandil. \\
\hline & If radiation related & $\begin{array}{l}\text { Do not biopsy } \\
\text { 1. Sucralfate enemas. } \\
\text { 2. Consider stool bulking/softening agent. } \\
\text { 3. Antibiotics. } \\
\text { 4. Consider hyperbaric oxygen therapy. } \\
\text { 5. Refer to a specialist centre. }\end{array}$ \\
\hline & $\begin{array}{l}\text { Mucosal prolapse/solitary } \\
\text { rectal ulcer }\end{array}$ & Refer to colorectal surgeon/gastroenterologist. \\
\hline & Neoplastic ulcer & $\begin{array}{l}\text { Refer urgently to appropriate oncology team requesting an } \\
\text { appointment within } 2 \text { weeks. }\end{array}$ \\
\hline
\end{tabular}

Gl, gastrointestinal; GTN, glyceril trinitrate.

\section{Steatorrhoea}

(the presence of excess fat in the stool)

\begin{tabular}{|c|c|c|}
\hline Investigations & Potential results & Clinical management plan: abnormal results \\
\hline \multicolumn{3}{|l|}{ 1st Line } \\
\hline Stool sample for faecal elastase & EPI & See EPI (page 16). \\
\hline Routine blood screen and additional blood screen & $\begin{array}{l}\text { Addison's disease Coeliac } \\
\text { disease } \\
\text { Thyroid dysfunction }\end{array}$ & $\begin{array}{l}\text { Follow treatment abnormal blood results } \\
\text { (pages } 2-3 \text { ). }\end{array}$ \\
\hline SeHCAT scan & BAM & Treatment for BAM (page 16). \\
\hline $\begin{array}{l}\text { Glucose hydrogen (methane) breath test for } \\
\text { bacterial overgrowth and/or OGD and D2 asp } \\
\text { and biopsies }\end{array}$ & $\begin{array}{l}\text { SIBO } \\
\text { Intestinal parasites }\end{array}$ & $\begin{array}{l}\text { Treatment for SIBO (page 17). } \\
\text { Treat with antibiotics within level of confidence or discuss with } \\
\text { microbiologists and supervising clinician. }\end{array}$ \\
\hline \multicolumn{3}{|l|}{ 2nd Line } \\
\hline $\begin{array}{l}\text { Gut hormones (Chromogranin A\&B, gastrin, } \\
\text { substance P, VIP, calcitonin, somatostatin, } \\
\text { pancreatic polypeptide) and Urinary 5-HIAA and } \\
\text { CT/MR liver and abdomen }\end{array}$ & $\begin{array}{l}\text { Pancreatic neuroendocrine } \\
\text { tumour }\end{array}$ & $\begin{array}{l}\text { Discuss and refer urgently to the appropriate neuroendocrine tumour } \\
\text { team, requesting an appointment within } 2 \text { weeks. }\end{array}$ \\
\hline \multirow[t]{2}{*}{$\begin{array}{l}\text { CT abdo pelvis/capsule endoscopy/MRI } \\
\text { enteroclysis }\end{array}$} & $\begin{array}{l}\text { Small intestinal disease other } \\
\text { than radiotherapy induced (eg, } \\
\text { lymphoma) }\end{array}$ & $\begin{array}{l}\text { Discuss immediately and refer urgently to the appropriate team requesting } \\
\text { an appointment within } 2 \text { weeks. }\end{array}$ \\
\hline & $\begin{array}{l}\text { If all tests are negative, but } \\
\text { symptoms persist }\end{array}$ & $\begin{array}{l}\text { 1. Trial of empirical antibiotics to exclude test negative SIBO (page 17). } \\
\text { 2. Trial of low fat diet. }\end{array}$ \\
\hline
\end{tabular}




\section{Tenesmus}

(a feeling of constantly needing to pass stools, despite an empty rectum)

\begin{tabular}{|c|c|c|}
\hline Investigations & Potential results & Clinical management plan: abnormal results \\
\hline Flexible sigmoidoscopy & $\begin{array}{l}\text { Radiation proctopathy } \\
\text { Anterior resection syndrome } \\
\text { Polyp } \\
\text { Newly diagnosed neoplasm } \\
\text { Newly diagnosed IBD/infection }\end{array}$ & $\begin{array}{l}\text { 1. Pelvic floor and toileting exercises (page 18). } \\
\text { 2. Stool bulking agent. } \\
\text { 3. Low dose antidepressants. } \\
\text { 4. Consider referral to a specialist centre for biofeedback (page 18). } \\
\text { 5. Consider referral for acupuncture. } \\
\text { Arrange endoscopic/surgical removal. } \\
\text { Refer urgently to the appropriate oncology team requesting an } \\
\text { appointment within } 2 \text { weeks. } \\
\text { 1. Send stool culture. } \\
\text { 2. If mild or moderate, refer within } 2 \text { weeks to a gastroenterologist. } \\
\text { If severe, this is an emergency-discuss immediately with a } \\
\text { gastroenterologist. }\end{array}$ \\
\hline
\end{tabular}

IBD, inflammatory bowel disease.

Weight loss (unexplained)

\begin{tabular}{|c|c|c|}
\hline Investigations & Potential results & Clinical management plan: abnormal results \\
\hline Dietary assessment & Inadequate dietary intake & $\begin{array}{l}\text { 1. Dietary advice and consider oral nutritional supplements. } \\
\text { 2. Refer for specialist dietetic assessment and advice. }\end{array}$ \\
\hline Symptom assessment & $\begin{array}{l}\text { No other Gl symptoms present } \\
\text { If Gl symptoms present }\end{array}$ & $\begin{array}{l}\text { 1. Discuss with supervising clinician or } \\
\text { 2. Request OGD, colonoscopy, CT chest, abdomen and pelvis. } \\
\text { Follow algorithm. }\end{array}$ \\
\hline $\begin{array}{l}\text { Routine and additional blood } \\
\text { screen (pages } 2-3 \text { ) and } \\
\text { myeloma screen }\end{array}$ & $\begin{array}{l}\text { Abnormal results, eg, } \\
\text { thyrotoxicosis, new onset } \\
\text { diabetes mellitus, Addison's } \\
\text { disease }\end{array}$ & Follow treatment of abnormal results (pages $2-3$ ). \\
\hline $\begin{array}{l}\text { OGD } \\
\text { Colonoscopy } \\
\text { CT chest abdomen and pelvis }\end{array}$ & $\begin{array}{l}\text { Organic cause (eg, infection, } \\
\text { inflammation, neoplastic) } \\
\text { No organic cause identified }\end{array}$ & $\begin{array}{l}\text { Discuss with supervising clinician within } 24 \mathrm{~h} \text {. } \\
\text { Refer to dietitian and review regularly. } \\
\text { Consider psychological causes, eg, depression/eating disorder. } \\
\text { Refer to appropriate cancer MDT for consideration of PET scan. }\end{array}$ \\
\hline
\end{tabular}

Gl, gastrointestinal; OGD, oesophago-gastroduodenoscopy; PET, position emission tomography.

Acknowledgements We sincerely thank the following experts who participated in the Delphi process and read and provided detailed feedback on this algorithm: Dr Ang Yeng, Mr Anthony Antoniou, Mrs Sharon Becker, Mr Neil Borley, Dr Stuart Cairns, Miss Helen Chave, Professor Susan Clark, Dr Susan Cleator, Dr Sue Cullen, Dr Tina Diggory, Dr NC Direkze, Ms Mhairi Donald, Dr Clare Donnellan, Dr Roland Ede, Dr Birgitte-Elise Grinde Emken, Ms Andreia Fernandes, Mr Nader Francis, Dr Lorenzo Fuccio, Dr Simon Gabe, Mrs Claire Gill, Mrs Loraine Gillespie, Dr Stephen Gore, Dr John Green, Dr Caroline Henson, Ms Linda Hill, Dr Barbara Hoeroldt, Ms Lynn Holmes, Mr Terry Irwin, Mr John (Ian) Jenkins, Mrs Penny Kaye, Dr Mark Kelly, Dr Simon Lal, Dr Susan Lalondrelle, Dr Jimmy K Limdi, Ms Michelle McTaggart, Dr Tracie Miles, Dr Michael Mitchell, Ms Kassandra Montanheiro, Dr A Frank Muller, Dr Andrew Murdock, Dr Penny Neild, Dr John O’Malley, Dr Parth Paskaran, Professor David Rampton, Dr Jeremy Shearman, Dr Norma Sidek, Ms Ramani Sitamvaram, Dr John Staffurth, Dr Alexandra Stewart, Dr Sreedhar Subramanian, Dr Claire Taylor, Dr Kathy Teahon, Dr Jeff Turner, Mrs Julie Walker, Dr Sean Weaver, Ms Sarah Wemyss, Dr Mark Wilkinson, Miss Natalie Wilkinson, Mrs Tracy Wood, Dr Jeremy Woodward. We would like to thank Dr Claire Dearden, Dr Mohid Khan and Dr Daniel Morganstein for additional helpful input into this document. We are very grateful to Barbara E. Benton for help with maintaining the integrity of early drafts of this guide.
Contributors All authors contributed to the study design. Algorithm development was performed by HJNA, ACM, CN and JOL. Guarantor of the article: HJNA.

Funding This study was undertaken at The Royal Marsden NHS Foundation Trust that received a proportion of its funding from the NHS Executive. We acknowledge NHS funding to the NIHR Biomedical Research Centre. Some of the work compiling this guide was facilitated by funding received from Macmillan Cancer Support.

Competing interests None.

Provenance and peer review Not commissioned; externally peer reviewed.

Open Access This is an Open Access article distributed in accordance with the Creative Commons Attribution Non Commercial (CC BY-NC 3.0) license, which permits others to distribute, remix, adapt, build upon this work noncommercially, and license their derivative works on different terms, provided the original work is properly cited and the use is non-commercial. See: http://creativecommons.org/licenses/by$\mathrm{nc} / 3.0 /$

\section{REFERENCES}

1 Walters J, Pattni S. Managing bile acid malabsorption. Therap Adv Gastroenterol 2010;3:349-57. 
2 Wedlake L, Thomas K, Lalji A, et al. Effectiveness and tolerability of colesevelam hydrochloride for bile acid malabsorption in patients with cancer: a retrospective chart review and patient questionnaire. Clin Ther 2009;31:2549-58.

3 Dookeran KA, Thompson MM, Allum WH. Pancreatic insufficiency secondary to abdominal radiotherapy. Eur J Surg Oncol 1993;19:95-6.

4 Kingham JG, Barrett A. Pancreatic insufficiency following abdominal irradiation. Postgrad Med J 1980;56:804-5.

5 Andreyev J. Gastrointestinal symptoms after pelvic radiotherapy: a new understanding to improve management of symptomatic patients. Lancet Oncol 2007;8:1007-17.

6 Suarez FL, Savaiano DA, Levitt MD. A Comparison of symptoms after the consumption of milk or lactose-hydrolyzed milk by people with self-reported severe lactose intolerance. N Engl J Med 1995;333:1-4.

7 Haylen B, de Ridder D, Freeman R, et al. An International Urogynaecological Association (IUGA)/International Continence Society (ICS) joint report on the terminology for female pelvic floor dysfunction. Neurol Urodyn 2010;29: 24-9.

8 Andreyev HJ, Davidson S, Gillespie C, et al. Practice guidance on the management of acute and chronic gastrointestinal problems arising as a result of treatment for cancer. Gut 2012;61:179-92.

9 Dukowicz A, Lacy B, Levine G. Small bowel bacterial overgrowth: a comprehensive review. Gastroenterol Hepatol 2007;3:112-22.

10 Gasbarrini A, Lauritano EC, Gabrielli M, et al. Small intestinal bacterial overgrowth: diagnosis and treatment. Dig Dis 2007;25:237-40.

11 Grace E, Shaw C, Whelan K, et al. Small intestinal bacterial overgrowth prevalence, clinical features, current and developing diagnostic tests, and treatment. Aliment Pharmacol Ther 2013;38:674-88.

12 Abayomi J, Kirwan J, Hackett A. Coping mechanisms used by women in an attempt to avoid symptoms of chronic radiation enteritis. J Hum Nutr Diet 2009;22:310-16.

13 Department of Health. National Diet and Nutrition Survey: Headline results from Years 1 and 2 (combined) of the rolling programme 2008/9-2009/10. 2011. http://www.dh.gov.uk/en/
Publicationsandstatistics/Publications/PublicationsStatistics/ DH_128166

14 Gami B, Harrington K, Blake P, et al. How patients manage gastrointestinal symptoms after pelvic radiotherapy. Aliment Pharmacol Ther 2003;18:987-94.

15 Wald A. Chronic constipation: advances in management. Neurogastroenterol Motil 2007;19:4-10.

16 National Institute of Health and Care Excellence. Management of faecal incontinence in adults: CG 49. London: NICE, 2007.

17 Law Y, Fielding J. MRI of pelvic floor dysfunction: review. AJR Online 2008;191:S45-53.

18 Rao SSC. Disorders of the Pelvic Floor and Anorectum. Gastorenterol Clin North Am 2008;37:493-506.

19 Sikirov D. Comparison of straining during defecation in three positions: results and implications for human health. Dig Dis Sci 2003;48:1201-5.

20 Cullen SN, Frenz M, Mee A. Treatment of haemorrhagic radiation-induced proctopathy using small volume topical formalin instillation. Aliment Pharmacol Ther 2006;23:1575-9.

21 Raman R. Two percent formalin retention enemas for haemorrhagic radiation proctitis: a preliminary report. Dis Col Rectum 2007;50:1-8.

22 Rubinstein E, Ibsen T, Rasmussen R, et al. Formalin treatment of radiation-induced hemorrhagic proctitis. Am J Gastroenterol 1986;81:44-5.

23 Botten RJ, Di Matteo AC, Butters J, et al. Randomised trial of argo plasma coagulation therapy versus topical formalin for persistent rectal bleeding. Int J Radiol Biol Phys 2013;87:954-9.

24 Nazarko L. Managing a common dermatological problem: incontinence dermatitis. Br J Community Nurs 2007; 12:358-63.

25 The Royal Marsden NHS Foundation Trust. The Royal Marsden Hospital Manual of Clinical Procedures. 8th edn. Dougherty L, Lister S. eds. Chapter 19. Wound Management: Radiotherapy skin reactions:1180-3.

26 Park MI, Camilleri M. Gastroparesis: clinical update. Am J Gastroenterol 2006;101:1129-39.

27 Khoo J, Rayner CK, Jones KL, et al. Pathophysiology and management of gastroparesis. Expert Rev Gastroenterol Hepatol 2009;3:167-81. 


\section{APPENDIX: COMMON CONDITIONS IN THIS GROUP}

Bile acid malabsorption (BAM)

Exocrine pancreatic insufficiency (EPI)

Carbohydrate malabsorption-for example, lactose or other disaccharide intolerance

Pelvic floor dysfunction

Small intestinal bacterial overgrowth (SIBO)

\section{BILE ACID MALABSORPTION}

Definition

BAM is a defect in the enterohepatic circulation of bile acids. Two types of BAM exist: ileal dysfunction whereby the ability to absorb bile acids in the terminal ileum is impaired and secondly, hepatic overproduction that overwhelms terminal ileal absorption capacity. ${ }^{1}$ Bile is secreted by the liver in direct response to the amount of ingested dietary fat.

Common causes:

- High dose chemotherapy

- Ileal disease/resection

- Upper GI resectional surgery including cholecystectomy

- Pancreatic disease

- Pelvic radiotherapy

- Idiopathic

Diagnosis:

- SeHCAT scan

- C4 blood test

- Trial of bile acid sequestrant

\section{SEVERITY SCORES OF BAM}

7 day SeHCAT retention BAM status

Treatment options include

\begin{tabular}{ll}
\hline $10-15 \%$ & Mild BAM \\
$5-10 \%$ & Moderate BAM \\
$\leq 5 \%$ & Severe BAM \\
\hline
\end{tabular}

1. dietary fat reduction

2. antidiarrhoeal medication

3. bile acid sequestrant

Options 1 and 2 may be useful in mild BAM. Generally bile acid sequestrants are required for moderate BAM. For severe BAM developing after radiotherapy, most patients need a bile acid sequestrant and advice about long-term reduction in dietary fat intake. $^{2}$

Drugs that may be helpful include aluminium hydroxide, budesonide, colesevelam, colestipol and colestyramine.

Patients with steatorrhoea usually require colesevelam.

If dietary intervention is required, advice to reduce dietary fat intake to $20 \%$ of total calories can be useful but requires dietetic expertise, patient education and supportive literature.
Many patients with moderate/severe BAM will be deficient in trace elements and fat-soluble vitamins. These should be checked periodically and supplemented as appropriate.

\section{EXOCRINE PANCREATIC INSUFFICIENCY}

\section{Definition}

EPI is the inadequate production and secretion of pancreatic enzymes and may occur after pelvic radiotherapy with para-aortic lymph node irradiation. ${ }^{34}$

Diagnosis:

Non-liquid stool sample for faecal elastase measurement $(<200 \mu \mathrm{g}$ FE1 per $1 \mathrm{~g}$ stool $)-$ N.B. Falsely low readings may be present in patients with small bowel bacterial overgrowth.

Clinical response to pancreatic replacement.

Treatment:

Pancreatic enzyme replacement therapy: requires equivalent of 150000 international units Creon (Abbott Healthcare Products Limited, Southampton, UK) per day.

- Optimal 30-50000 units with each meal, 10-30000 units with drinks and snacks, depending on size of snack.

- Consider long-term multivitamin and trace element supplementation.

- Occasionally dietary advice is also required to optimise bowel function.

- Occasionally addition of proton pump inhibitor is required to reduce loss of replacement enzymes by gastric acid.

Long-term management:

Ongoing treatment with pancreatic enzyme replacement medication.

\section{CARBOHYDRATE MALABSORPTION-FOR EXAMPLE, LACTOSE OR OTHER DISACCHARIDE INTOLERANCES}

\section{Definition}

Intolerance occurs from the inability to digest carbohydrates. Lactose, a component of milk and some other dairy products, is the intolerance most frequently recognised. It is due to lack of the enzyme lactase in the small intestine. Primary hypolactasia affects $70 \%$ of the world's population. Lactose or other disaccharide or monosaccharide malabsorption (eg, fructose) may occur de novo during cancer therapies (such as 5-FU chemotherapy or radiotherapy) due to damage to brush border enzymes and in some patients persists long term. ${ }^{5} 6$

Diagnosis of carbohydrate intolerance:

- Trial of exclusion of products containing that specific carbohydrate in diet for 1 week. Patient to keep a record of symptoms before and during the exclusion.

- Specific carbohydrate breath test. Duodenal biopsies and assessment for the specific disaccharide or monosaccharide activity. 
Treatment:

- Long-term exclusion of products containing the carbohydrate in diet.

- Dietitian assessment to ensure diet remains balanced. With lactose intolerance special attention should be paid to calcium intake. Other bone health risk factors should also be considered and vitamin and mineral supplementation started as appropriate. ${ }^{5}$

\section{PELVIC FLOOR DYSFUNCTION}

Definition

Symptoms of pelvic floor dysfunction include urinary incontinence, bladder storage problems, altered bladder sensation, voiding and post micturition problems, anorectal symptoms, pelvic pain, sexual difficulties and pelvic organ prolapse (in females). Anorectal symptoms can include faecal incontinence, flatal incontinence, faecal urgency, straining to defecate, tenesmus, diminished rectal sensation, constipation, rectal prolapse, rectal bleeding and mucus discharge. ${ }^{7}$

Diagnosis:

- rectal examination (sphincter tone and squeeze)

- endo-anal ultrasound

- anorectal physiology investigations:

- anal resting pressure

- sphincter muscle squeeze

- 15 s squeeze

- rectal sensitivity to rectal distension

Treatment:

- pelvic floor exercises (page 18)

- toileting posture exercises (page 18)

- biofeedback (page 18)

A contributing factor is often constipation and a non-fermentable stool bulking agent such as Normacol can be helpful to restore rectal volume and is less likely to cause flatulence than other fibre supplements.

\section{SMALL INTESTINAL BACTERIAL OVERGROWTH}

Definition

SIBO is the presence of excessive bacteria in the small intestine. Small bowel bacterial overgrowth occurs in $25 \%$ of patients during the acute phase of radiotherapy and is a cause of diarrhoea in up to $15 \%$ of patients after radiotherapy. 589

Diagnosis $^{10}$ :

- There is no gold standard for diagnosing SIBO.

- Glucose hydrogen/methane breath testing \pm duodenal (D2) aspirate via upper GI endoscopy.

- RBC folate and total serum bile acid levels may be elevated and vitamin B12 levels and faecal elastase may be low.

- $10-15 \%$ patients with negative tests still have SIBO. ${ }^{11}$

Suggested antibiotic treatment options if no growth on culture to direct treatment

7-10 days treatment with

- Ciprofloxacin $500 \mathrm{mg}$ bd
- Doxycycline $200 \mathrm{mg}$ day 1, $100 \mathrm{mg}$ days 2-7/10

- Clarithromycin $500 \mathrm{mg}$ bd

- Metronidazole $400 \mathrm{mg}$ tds

$>$ Rifaximin $550 \mathrm{mg}$ bd

Symptoms can recur any time after antibiotics are stopped because the underlying cause of bacterial overgrowth cannot always be addressed. If symptoms return, repeat treatment with antibiotics for a few days every month or continually at the lowest effective dose may be helpful in managing symptoms long term. Some clinicians recommend rotating antibiotics, but this may not be effective if the organisms involved are not sensitive to the antibiotics used.

Treatment decisions should be individualised and consider the risks of long-term antibiotic therapy such as Clostridium difficile infection, cumulative irreversible neuropathy with metronidazole, Achilles tendon rupture with ciprofloxacin, intolerance, side effects, bacterial resistance and costs. ${ }^{8-11}$

\section{APPENDIX: \\ MANAGEMENT TECHNIQUES}

Written information is often helpful to supplement the management of specific diagnoses. If information sheets are not available locally, information sheets on the following can be obtained from Dr Andreyev's office at The Royal Marsden (+44 (0)20 7811 8216):

1. advice for those with constipation or who often need to strain

2. having a SeHCAT scan

3. having a glucose hydrogen/methane breath test

4. pancreatic insufficiency.

Specific leaflets are also available on the following treatments:

1. lactose free diet

2. managing fibre in your diet

3. taking antidiarrhoeal medication

4. taking colesevelam

5. taking loperamide

6. taking Normacol

7. treatment of radiation-induced gastrointestinal bleeding.

\section{DIETARY FIBRE MANIPULATION}

The mean UK average consumption of non-starch polysaccharides for healthy adults (aged 19-64 years) is $14.9 \mathrm{~g} /$ day for men and $12.8 \mathrm{~g} /$ day for women. ${ }^{12}$ The current dietary recommended daily intake for fibre-18 g non-starch polysaccharides per day-is based on the effect that total dietary fibre has on stool weight. The rationale for this is that daily stool output of $<100 \mathrm{~g} /$ day is associated with a non-starch polysaccharides intake of below $12 \mathrm{~g} /$ day and with an increased risk of bowel disease. In healthy populations, increasing non-starch polysaccharides intake from 13 to $18 \mathrm{~g} /$ day is associated with a $25 \%$ increase in stool weight. Some patients after pelvic radiotherapy cannot tolerate as much fibre as this. 
Reduction in soluble dietary fibre intake can be helpful when patients complain of any of the following symptoms: bloating, constipation, bowel obstruction, diarrhoea, rectal flatulence, mucus discharge and abdominal pain and may require help from a dietitian. ${ }^{12-14}$

\section{BIOFEEDBACK}

Biofeedback is widely regarded as a useful, noninvasive treatment in constipation, evacuatory disorders and faecal incontinence. Biofeedback is a behavioural approach to which there are no side effects and offers a non-surgical approach for patients with bowel dysfunction. It includes toileting exercises and pelvic floor exercises. ${ }^{15} 16$

Biofeedback services are available locally around the UK.

\section{PELVIC FLOOR EXERCISES}

The pelvic floor muscles include the levator ani, the coccygeus and associated connective tissue and, if weakened, can cause several symptoms associated with pelvic floor dysfunction (page 17). Exercises can strengthen the pelvic muscles so that they give support and are better coordinated. Pelvic floor exercises can improve problems with urinary incontinence, faecal incontinence or leakage and sexual function. ${ }^{17} 18$

Technique:

1. Sit, stand or lie with your knees slightly apart. Tighten and pull up your bottom muscles as tightly as you can. Hold for at least $5 \mathrm{~s}$ and then relax for at least $10 \mathrm{~s}$. Repeat at least five times. This will work on the strength of your muscles.

2. Next, pull the muscles up to about half of their maximum squeeze. See how long you can hold this for. Then relax for at least $10 \mathrm{~s}$. Repeat at least five times. This will work on the endurance or staying power of your muscles and will improve their coordination.

3. Pull up the muscles as quickly and tightly as you can and then relax and then pull up again, and see how many times you can do this before you get tired. Try for at least five quick pull-ups.

4. Repeat exercises 1,2 and 3 at least 10 times every day.

5. As the muscles get stronger, you will find that you can hold for longer than $5 \mathrm{~s}$, and that you can do more pull-ups each time without the muscle getting tired.

Patient information:

http://www.yourpelvicfloor.co.uk/

\section{TOILET POSTURE EXERCISES}

Adopting the correct position on the lavatory can improve constipation and ease evacuation difficulties together with pelvic floor exercises. ${ }^{19}$

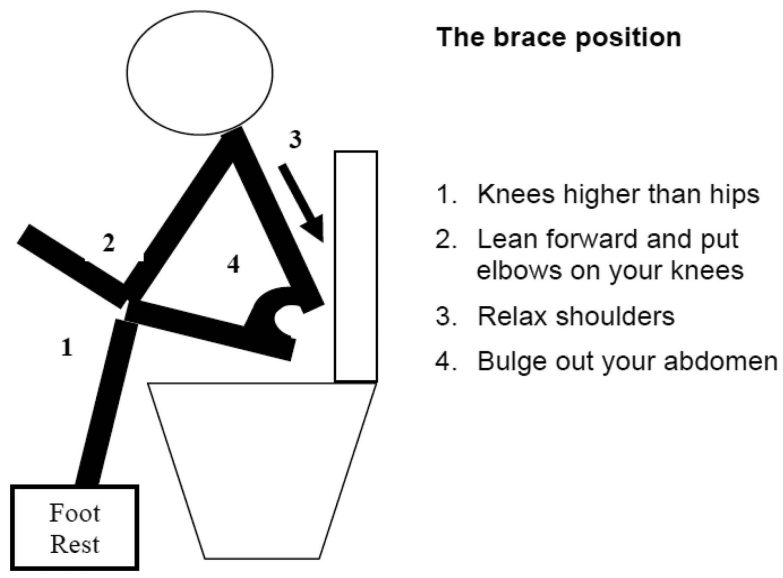

Technique

1. Sitting on the toilet, lean forward with the forearms resting on your thighs and raise your feet $8-10$ inches off the floor.

2. Relax and lower the shoulders. Breathe slowly, regularly and gently-do not hold your breath as this will encourage straining. Try and stay as relaxed as possible

3. Try and brace your abdominal muscles. This is best done by putting your hands on your waist. Expand your waist and feel your hands being pushed out sideways. Concentrate on relaxing your anus to allow the stool to pass. Only push down from above once your anus is relaxed.

4. Relax very slightly for $1 \mathrm{~s}$ maintaining pressure but without the push.

5. Then brace outwards and push down again.

6. Repeat steps 1-5.

Advise the patient to be careful to relax and use the correct technique, not to spend endless time in the toilet and not to strain-try again the next day. Excessive straining uses the wrong muscles and does not help the evacuating process.

\section{TREATING BLEEDING TELANGIECTASIA}

Radiation-induced bleeding typically starts 612 months after radiotherapy, is at its worse 4 years after the end of radiotherapy and has disappeared by $8-10$ years.

1. Investigate with flexible endoscopy to determine the cause of the bleeding.

2. Optimise bowel function and stool consistency.

3. If bleeding is not staining clothes, causing anaemia or interfering with daily activities, reassure and do nothing.

4. If bleeding affects quality of life, stop anticoagulants if possible and consider sucralfate enemas \pm metronidazole $400 \mathrm{mg}$ tds for 4 weeks.

5. Discuss definitive treatment to ablate the telangiectasia:

a. Hyperbaric oxygen therapy: Advantages: supported by RCT evidence, may improve other symptoms, for example, urinary; disadvantages: time consuming 
(8 weeks of daily treatment), expensive and patients may need to travel long distances to their nearest unit.

b. Any thermal therapy (eg, APC): Advantages: easily available and simple; disadvantages: significant risk of non-healing ulceration/perforation as the tissue is ischaemic and unproven efficacy in heavy bleeding. Absolutely contraindicated in patients treated with brachytherapy. If used, the bowel must be fully prepared (as for colonoscopy).

c. Formalin therapy: Advantages: simple to perform; disadvantages: long-term outcomes poorly known, small risks of serum sickness, severe proctitis or chemical burn to the skin if there is spillage.

\section{USING INTRARECTAL FORMALIN FOR RADIATION INDUCED TELANGIECTASIA}

Formalin chemically cauterises by hydrolysing protein and superficially coagulating the tissue. In general, the procedure seems to be effective, safe, well tolerated by the patient, inexpensive and technically simple. The use of intrarectal formalin for radiation-induced bleeding is contraindicated if the rectal mucosa is ulcerated. ${ }^{8}$ 20-23

Preparation:

- Prepare the bowel as for colonoscopy with full bowel preparation.

- Use a gastroscope not a colonoscope.

- $30-35 \mathrm{~mL}$ of $5 \%$ formalin is usually sufficient to cover the telangiectasia.

- Initial instillation of saline can help assess how much formalin will be required.

Technique:

- Patient position: prone.

- Instil the formalin through a catheter passed through the gastroscope channel into the rectum.

- Apply with wet cloths and continued pressure to the perianal area to prevent leakage of formalin during the procedure (by endoscopy nurse).

- Keep the gastroscope in place during the procedure.

- Leave the formalin in the rectum for 3 min exactly.

- Then remove the formalin with copious washes of water.

- Continue the perianal pressure/pads until all of the rectal formalin removed.

Considerations after treatment:

- Advise patients they may not notice any improvement in bleeding for 1-2 weeks.

- Consider the use sucralfate enemas for 2-3 weeks twice daily to help healing.

- Consider retreating if necessary 6-8 weeks later and repeat if necessary a third time after a further $6-8$ weeks.

\section{HOW TO REFER FOR HYPERBARIC OXYGEN THERAPY}

Hyperbaric oxygen for radiation-induced damage requires a funding application to the Specialised Commissioning Group. The local hyperbaric oxygen unit and the referring clinician need to fill in the application jointly. ${ }^{8}$

Hyperbaric units that provide medically supervised hyperbaric oxygen therapy with the correct pressures believed to be useful for treating radiation injury are only found at the following centres:

- DDRC Hyperbaric Medical Centre, Tamar Science Park, Plymouth

- James Paget Hospital, Great Yarmouth

- Midlands Diving Chamber, Hospital of St Cross, Rugby

- Spire Hospital, Cardiff

- Spire Hull and East Riding Hospital, Hull

- Spire Murrayfield Hospital, Wirral

- St John and Elizabeth Hospital, North London

- St Richards Hospital, Chichester

- Whipps Cross Hospital, East London

There is no evidence at all that non-medically supervised hyperbaric oxygen therapy as provided by MS treatment centres is of any benefit for treating radiation-induced toxicity.

\section{SUCRALFATE ENEMAS}

Sucralfate forms a mechanical protective layer over radiation-induced telangiectasia and improves healing.

1. Use $2 \mathrm{~g}(10 \mathrm{~mL})$ sucralfate suspension $(1 \mathrm{~g}$ in $5 \mathrm{~mL})$ made up to $50 \mathrm{~mL}$ using warm tap water in a bladder syringe.

2. Attach a soft, lubricated Foley catheter to the syringe.

3. The patient should insert the catheter gently into their rectum and instil the enema twice a day until the bleeding has stopped.

4. The enema should be held in the rectum as long as possible.

5. The patient should roll over at least once to coat the entire rectum but spend the majority of the time lying prone so the solution treats the anterior rectal wall.

6. Long-term once-daily enemas may help prevent the bleeding recurring.

7. If bleeding starts again, go back to using sucralfate enemas twice a day.

\section{PERIANAL SKIN CARE}

Radiotherapy skin reactions may present as skin irritation, erythema and ulceration and atrophy of the skin within the radiotherapy field that may be worsened by enzymes present in faecal fluid when incontinent or leaking.

Key principles: ${ }^{24} 25$

- Keep skin dry

- Keep skin free of faeces

- Prevent the development of perianal dermatitis by:

- Use 'simple' soap or 'Dove Sensitive' soap that will not affect the $\mathrm{pH}$ of the skin (normally 5.5). Regular soap has a $\mathrm{pH}$ of nine and can disrupt the skin $\mathrm{pH}$, which inhibits the growth of bacteria and thus increases dermatitis.

1. Treating the underlying cause:

- Use a skin barrier: cream or film. 
- Please note whether the patient has any allergies to any of the constituents.

- If the skin is damaged, Cavilon No Sting Barrier Film (3M, Loughborough, UK) or Epaderm (Molnlycke Health Care Group, Dunstable, UK) can be very useful.

- Consider the use of antifungal creams or corticosteroids (for a short period only).

- Consider referral for specialist dermatology assessment and advice.

Patient information:

http://www.stmarkshospital.org.uk/patientinformationleaflets

\section{USING PROKINETICS}

Effects on stomach: ${ }^{26} 27$

- Erythromycin: largely ineffective after 4-8 weeks through tachyphylaxis. Recommended dose $250 \mathrm{mg}$ bd as a syrup $30 \mathrm{~min}$ before food.
- Domperidone: no tachyphylaxis for 8 weeks, may occur after longer use. Recommended dose $10 \mathrm{mg}$ qds $30 \mathrm{~min}$ before food as a syrup orally or $30 \mathrm{mg}$ qds as a rectal suppository. Increased risk of cardiac arrhythmia.

- Metoclopramide: risk of tardive dyskinesia with use $>3$ months.

- Naloxone by subcutaneous infusion.

- Paroxetine that stimulates SI motility only.

\section{HOW TO PERFORM A DUODENAL ASPIRATE}

Treatment:

1. Flush $100 \mathrm{~mL}$ of sterile saline into the duodenum via the endoscope channel.

2. Follow this by $20 \mathrm{~mL}$ of air to ensure no saline remains in the endoscope channel.

3. Turn down the suction.

4. Leave the fluid to equilibrate with the duodenal contents for $10-20 \mathrm{~s}$.

5. Aspirate $20 \mathrm{~mL}$ of fluid into a sterile trap.

6. Send the duodenal aspirate sample directly to microbiology. 